\title{
Secretory microRNA Profiles of Third- and Fourth-Stage Dirofilaria immitis Larvae with Different Macrocyclic Lactone Susceptibility: In Search of Biomarkers for Early Detection of Infection
}

\author{
Lucienne Tritten ${ }^{1, *,+}+\mathbb{D}$, Erica J. Burkman ${ }^{2, t, \ddagger}$, Tobias Clark $^{3}$ and Guilherme G. Verocai ${ }^{4, *}$ \\ 1 Institute of Parasitology, University of Zurich, 8057 Zurich, Switzerland \\ 2 Department of Infectious Diseases, College of Veterinary Medicine, University of Georgia, \\ Athens, GA 30602, USA; erica.burkman@boehringer-ingelheim.com \\ 3 Veterinary Medicine Research and Development, Zoetis Inc., 333 Portage Street, Kalamazoo, MI 49007, USA; \\ tobias.clark@zoetis.com \\ 4 Department of Veterinary Pathobiology, College of Veterinary Medicine and Biomedical Sciences, Texas A\&M \\ University, College Station, TX 77843, USA \\ * Correspondence: lucienne.tritten@uzh.ch (L.T.); gverocai@cvm.tamu.edu (G.G.V.); Tel.: +41-44-635-8501 (L.T.); \\ +1-979-862-4999 (G.G.V.) \\ + These authors contributed equally to this work. \\ $\ddagger$ Current address: Global Innovation Parasitology, Boehringer Ingelheim Animal Health, 1730 Olympic Drive, \\ Athens, GA 30601, USA.
}

check for updates

Citation: Tritten, L.; Burkman, E.J.; Clark, T.; Verocai, G.G. Secretory microRNA Profiles of Third- and Fourth-Stage Dirofilaria immitis Larvae with Different Macrocyclic Lactone Susceptibility: In Search of Biomarkers for Early Detection of Infection. Pathogens 2021, 10, 786. https://doi.org/10.3390/pathogens 10070786

Academic Editor: Hans-Peter Fuehrer

Received: 28 May 2021

Accepted: 18 June 2021

Published: 22 June 2021

Publisher's Note: MDPI stays neutral with regard to jurisdictional claims in published maps and institutional affiliations.

Copyright: () 2021 by the authors. Licensee MDPI, Basel, Switzerland. This article is an open access article distributed under the terms and conditions of the Creative Commons Attribution (CC BY) license (https:// creativecommons.org/licenses/by/ $4.0 /)$.
Abstract: The canine heartworm, Dirofilaria immitis, is among the most important parasites of dogs in the United States and worldwide, and may cause severe and potentially fatal disease. Current diagnostic recommendations rely on serological detection of an adult female antigen, and visualization of microfilariae in the blood. Therefore, a reliable diagnosis can be only performed approximately six months post-infection. There is a growing need to characterize novel diagnostic markers that are capable of detecting the early stages of heartworm infection, in special markers associated with third-stage larvae (L3) and fourth-stage larvae (L4). The early detection of infection would guide medical interventions that could impede the development of patent infections and further parasite transmission. We cultured D. immitis L3 and L4 of two laboratorial strains with different susceptibility statuses to macrocyclic lactone drugs in vitro. Excretory/secretory microRNAs were sequenced and analyzed. We identified two miRNA novel candidates secreted abundantly by both L3 and L4 of both strains. These candidates were previously detected in the secretions of other D. immitis stages and one of them was found in the blood of D. immitis-infected dogs. These miRNAs have not been found in the secretions of other nematodes and could be D. immitis-specific diagnostic biomarkers, which could allow for the early detection of infection.

Keywords: diagnostic biomarkers; Dirofilaria immitis; heartworm; microRNA; early diagnosis; drug resistance; macrocyclic lactones; ivermectin

\section{Introduction}

The canine heartworm, Dirofilaria immitis, is arguably the most important parasite of dogs in the United States of America (USA) and across many countries worldwide. Heartworm is known to cause severe and often fatal disease in infected dogs, cats, and ferrets [1,2]. In addition to being the most prevalent filarial parasite of dogs in the country, heartworm incidence has been increasing in recent years, particularly in the southeastern United States [3].

The current guidelines of the American Heartworm Society recommend annual testing of all dogs in order to ensure the achievement and maintenance of successful prophylaxis. This recommendation includes the concurrent use of both an antigen detection test and a 
microscopy-based microfilariae ( $\mathrm{mf}$ ) detection test [4]. Currently, commercially available immunodiagnostic assays for the detection of heartworm antigens are used widely by practicing veterinarians and diagnostic laboratories. Such antigen detection tests target proteins secreted by mature adult $D$. immitis females. Despite their high sensitivity and specificity [4,5], a diagnosis can be only achieved approximately six months post-infection, which coincides with the average pre-patent period of heartworm.

There is a growing need to explore and characterize novel heartworm biomarkers that are capable of detecting the early stages of infection, that is, infections that are less than six months old. Earlier infections can be characterized by the presence of third-stage larvae (L3) and fourth-stage larvae (L4) and/or young adults (L5) within the organism of a definitive host, and the absence of sexually mature adult female and male worms in the hosts' pulmonary arteries [6]. An early diagnosis of heartworm infection in conjunction with appropriate clinical management could be beneficial for the health and wellbeing of the infected individual and beyond by impeding the development of patent infections. As a consequence, fewer dogs would become reservoirs for infection of suitable Culicidae mosquito vectors, impacting heartworm transmission at a local scale. In addition, dogs would be less likely to progress into severe and/or fatal clinical heartworm disease.

Helminth parasites, particularly, have evolved sophisticated mechanisms of communication with their hosts. Various molecules secreted or excreted by helminths have been shown to participate in these processes $[7,8]$. Among them are microRNAs (miRNAs), short non-coding RNA molecules of 18-25 nucleotides in length, with important posttranscriptional regulatory functions of gene expression. The presence and detectability of nematode-specific miRNAs in hosts' biofluids make them attractive diagnostic biomarker candidates for a wide range of diseases, and require minimal invasion [9]. To date, a handful of studies described the secreted miRNA profiles of various filarial nematodes, including D. immitis [9-13]. Such parasitic nematode-derived miRNAs have been found circulating in biological fluids (e.g., serum, plasma) and tissues of naturally infected hosts. The acknowledged presence of specific parasitic nematode-derived miRNAs in host serum and plasma highlights their potential as biomarkers of infection by filarioid species of veterinary and public health relevance [14,15].

Previously, D. immitis-derived miRNAs have been characterized in the plasma of dogs with patent experimental infections (therefore, with the concomitant presence of $\mathrm{mf}$ and adults) [9]. In addition, it was shown that some D. immitis-derived secretory miRNAs are differentially expressed across developmental stages or sexes of adults, highlighting their potential as markers for detecting the early stages of heartworm infection [16]. Additionally, nematode-derived miRNAs have been detected in the blood of animals and humans infected with tissue-dwelling filarial nematodes such as Onchocerca volvulus, O. ochengi, and Loa loa, which, similarly to D. immitis L3 and L4, do not reside in the circulatory system $[10,12,17]$.

In order to assess the potential of D. immitis-derived miRNAs as diagnostic candidates in vivo, various elements remain to be clarified. In this study, we aimed to assess whether selected $D$. immitis L3 and/or L4-derived miRNAs in vitro may be biomarker candidates for the early detection of infection, both in terms of abundance and species specificity, as well as the consistency of stage-specific miRNA secretion across replicates and $D$. immitis laboratorial strains with different susceptibilities to macrocyclic lactone drugs. We compared our results to the existing literature and identified two novel miRNA candidates secreted abundantly by both L3 and L4 developmental stages and in both strains.

\section{Results}

\subsection{Libraries and General Results}

Six samples from each strain, Yazoo (resistant to macrocyclic lactones) and Missouri (susceptible to macrocyclic lactones) were analyzed, encompassing three replicates from L3, and three from L4. Libraries contained between 5.49 and 10.86 million reads. Across all samples (i.e., strains and stages), a total of 187 unique mature miRNAs with different 
degrees of confidence were discovered (Supplementary Table S1). For the subsequent analyses, we stratified the data by strain and/or stage (Supplementary Tables S2-S5).

\subsection{Strain and Stage Specificity}

We considered all miRNA candidates for our initial analysis, including those with no known orthologues among nematode sequences available in miRBase. We identified a total of 187 mature miRNA sequences across all six samples (Supplementary Table S1). The level of agreement between both strains and the two stages was high. Several members of the miR-100 family were among the top 10 most abundantly represented sequences, as well as lin-4 and let-7 (Table 1). Bma-miR-81, bma-miR-57, and bma-miR-92 were also among the highly abundant sequences present in all samples (if not among the top 10).

Differential expression analysis was performed, taking all miRNAs into account expressed in at least one sample (Figure 1). One single miRNA (bma-miR-87a_R+1) was consistently found in L4 sample replicates in low levels (between 11 and 15 copies, normalized counts), but not in L3 samples of the strain Yazoo. The trend was similar in the strain Missouri, but the expression in both examined stages was not significantly different. bma-miR-87a_R+1 was always present in L4 secretions, found between 5 and 60 copies, and between 0 (in 2 samples) and 9 copies in L3 samples (Missouri, normalized counts). One miRNA (bma-miR-228) was found to be more abundant in Missouri than in Yazoo L3 products (normalized counts; Figure 1); it was, however, present in all replicates of both stages. Similarly, PC-5p-7293_584 was present in all samples and replicates were in high amounts and detected in slightly higher copy numbers in Missouri than in Yazoo L4 supernatants.

There were 2 predicted candidates (PC-5p-7293_584 and PC-3p-5433_825) that were also highly and consistently represented in all 4 samples (L3s and L4s of both strains) and are among the 20 most abundant miRNAs. These miRNAs map to the $D$. immitis genome but have no described orthologues among the available nematode miRNAs in miRBase v21. Hence, these might represent miRNAs specific to D. immitis. PC-5p-7293_584 was the 7th and 9th most abundant miRNA in L3 supernatants from the Yazoo and Missouri strains, respectively (191 and 393 copies), while it ranked 15th and 16th most abundant in L4 secretions, from the Yazoo and Missouri strains, respectively (>100 copies).

PC-3p-5433_825 was the 9th and 13th most abundant miRNA in L3 supernatants from the Yazoo and Missouri strains ( $>100$ copies), respectively, while it ranked 10th and 12 th most abundant in L4 from the Yazoo and Missouri strains, respectively (>200 copies); details are available in Supplementary Tables S2-S5.

\subsection{Comparison to Previous Reports}

At the sequence level, and taking all miRNAs into account regardless of the confidence status, 26 miRNAs were common to L3 secretions from the current study and an initial prior study conducted in vitro [16]. Similarly, 39 sequences were found in cultures from L4s in both studies, with both strains combined (Figure 2). In miRNA discovery experiments, it is likely that a fraction of predicted candidate sequences do not correspond to real miRNAs but rather to artifacts; therefore, these numbers must be interpreted with caution. Despite the fact that the same species and stages were examined in the compared studies, only a small fraction of miRNA sequences overlapped. Here, whole mature sequences were considered, despite the fact that the $3^{\prime}$ end of mature sequences are known to be subject to variation [18]. 
Table 1. Top 10 most abundant miRNAs. The ranking was established based on differential expression analysis for Yazoo and Missouri separately.

\begin{tabular}{|c|c|c|c|c|c|c|}
\hline \multicolumn{5}{|c|}{ Top 10 Most Abundant miRNAs } & \multicolumn{2}{|c|}{ Previous Study [16] } \\
\hline & Yazoo L3 & Missouri L3 & Yazoo L4 & Missouri L4 & L3 & L4 \\
\hline 1 & bma-miR-100a_R+1 ** & bma-miR-100a_R+1 ** & bma-miR-100a_R+1 ** & bma-miR-100a_R+1 ** & bma-lin-4 & bma-lin-4 \\
\hline 2 & bma-miR-100b_R+2 * & bma-miR-100b_R+2 * & bma-lin- 4 * & bma-lin- $4^{* *}$ & PC-3p-389_2939 & bma-miR-92_R+2 \\
\hline 3 & bma-lin- 4 * & bma-lin- 4 * & bma-miR-100b_R+2 * & bma-miR-100b_R+2* & asu-let-7-5p & asu-let-7-5p \\
\hline 4 & bma-miR-100d_R+1 * & bma-miR-100d_R+1 * & bma-miR-100d_R+1 * & bma-miR-100d_R+1 * & bma-miR-100b_R+1 & bma-miR-100a_R+1 \\
\hline 5 & asu-let-7-5p & asu-let-7-5p & bma-miR-81_R+1 & bma-miR-81_ēR+1 * & bma-miR-100a_R+1 & bma-miR-100b_R+1 \\
\hline 6 & $\begin{array}{c}\text { bma-miR- } \\
\text { 100c_R+1_1ss12CT }\end{array}$ & bma-miR-81_R+1 & $\begin{array}{c}\text { bma-miR- } \\
\text { 100c_R+1_1ss12CT }\end{array}$ & bma-miR-57 & bma-miR-92_R+2 & bma-miR-57 \\
\hline 7 & PC-5p-7293_584 & $\begin{array}{c}\text { bma-miR- } \\
\text { 100c_R+1_1ss12CT }\end{array}$ & bma-miR-57 & bma-miR-92_R+2 & bma-miR-100d_R+1 & bma-miR-100d_R+1 \\
\hline 8 & bma-miR-57 & bma-miR-57 & bma-miR-92_R+2 & $\begin{array}{c}\text { bma-miR- } \\
\text { 100c_R+1_1ss12CT }\end{array}$ & bma-miR-5364 & PC-3p-389_2939 \\
\hline 9 & PC-3p-5433_825 & PC-5p-7293_584 & asu-let-7-5p & bma-miR-239-5p & bma-miR-228 & bma-miR-5364 \\
\hline 10 & bma-miR-81_R+1 & bma-miR-7_R+1_1ss10TA & PC-3p-5433_825 & bma-miR-71_R+4 & hco-miR-9 & $\begin{array}{c}\text { bma-bantam_R+1 } \\
(=\text { bma-miR-81_R+1) }\end{array}$ \\
\hline
\end{tabular}

${ }^{* *}$ present in $>10,000$ copies (normalized counts); ${ }^{*}$ present in $>1000$ copies (normalized counts). 'Predicted candidates' of interest are bolded. 
A

\section{L3 vs L4}

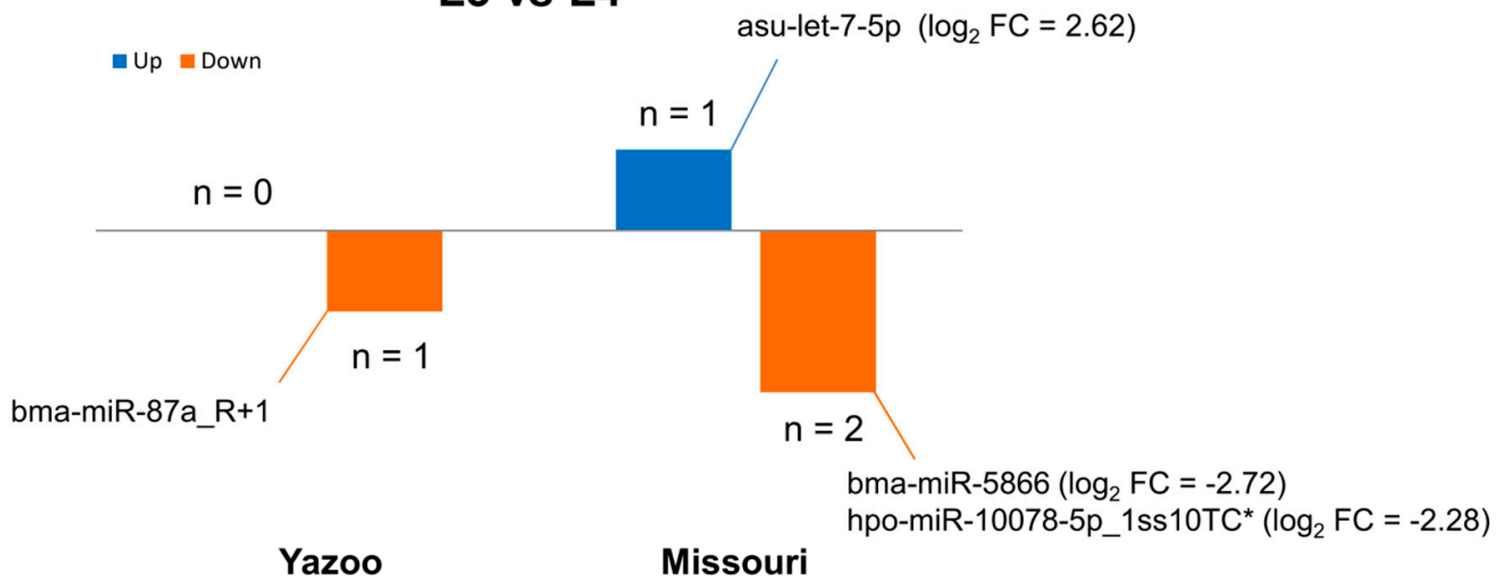

B

\section{Yazoo vs Missouri}

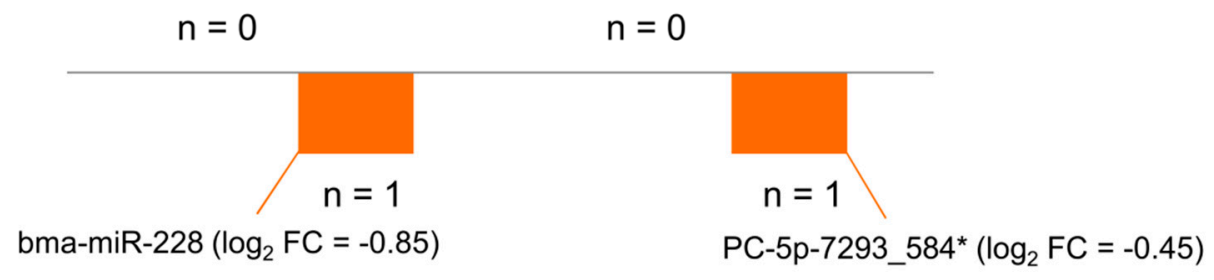

L3

L4

Figure 1. The number of differentially expressed miRNAs across pairwise comparisons. The differential expression was stratified by stages and strain. (A) Differentially expressed miRNAs between stages. For the Yazoo strain, bma-miR-87a_R+1 was absent in L3 secretions (hence, no fold-change). In the Missouri strain, asu-let-7-5p was more abundant in L3 compared to L4, and two sequences were less abundant in L3 compared to L4 (bma-miR-5866 and hpo-miR-10078-5p_1ss10TC). (B) Differentially expressed miRNAs between D. immitis strains. Bma-miR-228 was less abundant in Yazoo L3 (resistant to macrocyclic lactones) compared to Missouri L3 (susceptible to macrocyclic lactones); PC-5p-7293_584 was less abundant in Yazoo L4 compared to Missouri L4. * indicates 'low confidence' sequences, requiring validation. $\log 2 \mathrm{FC}=\log 2$ fold change in pairwise comparisons. All are statistically significant with a set $p$-value $<0.05$.

A very similar miRNA ranking by abundance had been shown in the supernatants of cultured L3 and L4, especially when extended beyond the top 10 shown in Table 1 [16]. A sequence similar to PC-5p-7293_584 was found in that previous report, under the name PC-5p-263_4142, and it was the 23rd and 32nd most abundant among L3 and L4 secretions, respectively. Similarly, PC-3p-5433_825 was found under the name PC-3p-955_1342 in the previous paper [16], and it was the 24th and 26th most abundant in L3 and L4 secretions, respectively. Both sequences were also released abundantly by $\mathrm{mf}$, adult males, and females; however, PC-3p-5433_825 was slightly more abundant in the secretions of L3 and L4 in normalized count libraries (Table 2). However, these were not reported in the D. immitis somatic miRNAome [19], and have so far not been reported in the secretions of other filarial nematodes in vivo (in host blood). Sequence alignments with previously reported similar sequences are shown in Figure 3. 
L3

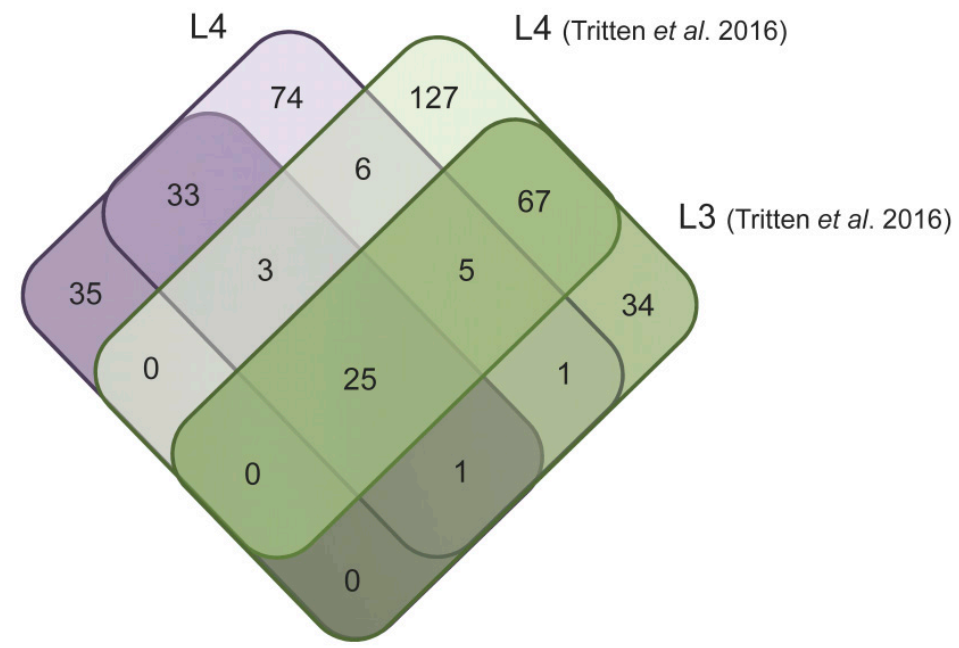

Figure 2. Venn diagram showing miRNAs common to datasets. Comparison of mature miRNA sequences across L3 and L4 secretions with a previous study [16]. Both strains of the current study were considered, as well as 'low confidence' sequences (e.g., not listed in miRBase), as they may include novel sequences.

Table 2. Literature reportings of two predicted candidates in D. immitis and other filarial nematodes. Other filarial species included Onchocerca ochengi, Loa loa, O. volvulus, and Litomosoides sigmodontis * (studies based on host blood; [9,10,12,17]).

\begin{tabular}{ccccc}
\hline & In Supernatants? [16] & $\begin{array}{c}\text { In Infected Dog } \\
\text { Blood? [9] }\end{array}$ & $\begin{array}{c}\text { In D. immitis Somatic } \\
\text { miRNA Pool? [19] }\end{array}$ & $\begin{array}{c}\text { In Other Filarial } \\
\text { Species? * }\end{array}$ \\
\hline PC-5p-7293_584 & Yes & No & No & No \\
PC-3p-5433_825 & Yes & Yes & No & No \\
\hline
\end{tabular}

A)

\begin{tabular}{|c|c|}
\hline$P C-5 p-7293 \quad 584$ & -CCTGAGCAGGATGAAGCC \\
\hline$P C-5 p-263-4142$ & 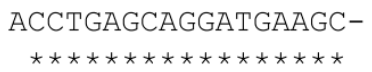 \\
\hline
\end{tabular}

B)

PC-3p-5433 825

$\mathrm{PC}-3 \mathrm{p}-955 \overline{1} \mathbf{1 3 4 2}$

$\mathrm{PC}-3 \mathrm{p}-2144 \quad 333$

PC-3p-2034_418
GAACCTCACCTCGGGCACCAGA-

GAACCTCACCTCGGGCACCAGAA

GAACCTCACCTCGGGCACCAGAA

GAACCTCACCTCGGGCACCAGAA

$\star * * * * * * * * * * * * * * * * * * * * *$

Figure 3. Sequence alignments of predicted candidates found in this study and previous reports. Sequences described in the current study are indicated in bold and red. (A) Alignments with PC-5p7293_584. (B) Alignments with PC-3p-5433_825. Stars represent nucleotide matching positions in the predicted mature sequence. PC-5p-263_4142 and PC-3p-955_1342 were detected in vitro [16], while PC-3p-2144_333 and PC-3p-2034_418 were previously described in infected dogs (laboratory and natural infections) [9].

In D. immitis-infected dog serum, bma-miR-87a_R+1 was the 9th most abundant [9] and found in supernatants from all stages examined [16]. The sequence of PC-3p-5433_825 was found among the top 20 in dog serum as well (under the name PC-3p-2144_333), in a pool of samples from laboratory infections (from both Missouri and MP3 strains), as well as in a natural infection (under the name PC-3p-2034_418) [9] (Figure 3). 


\subsection{Target Prediction in Host Genome and Overrepresentation Analysis}

In an attempt to understand the biological significance of the extracellular presence of these miRNAs in the canine host, computational target prediction within the dog transcriptome was conducted. Target prediction informs on the putative biological functions that D. immitis larvae may regulate in the dog by the secretion of miRNAs. Considering 'highconfidence' miRNAs (Groups 1, 2a, 2b; see Materials and Methods and Supplementary Table S1) and predicted candidates (Group 4), present in at least one sample in 10 copies or more, $58 \mathrm{~L} 3$ miRNAs and $94 \mathrm{~L} 4$ miRNAs were used for a computational target prediction within the dog genome, using the stand-alone TargetScan algorithm. Over 15,000 dog genes appeared to be targeted by the selected miRNAs from each stage. Overrepresentation analysis was used to identify the most significantly targeted host pathways within immune functions. The top 5 enriched pathways based on FDR are listed in Table 3. There were 84 categories significantly enriched in the predicted targets of L3 miRNAs. Behind several of the gene ontology (GO) descriptions are many fibroblast growth factor (FGF) isoforms, FGF receptors, as well as forkhead box proteins, among many others (146 in total). There were 64 categories significantly enriched in the predicted targets of L4 miRNAs. Generally, membrane transport features are predicted to be impacted by L4-secreted miRNAs. Interestingly, MAPK cascade was found among the top enriched categories.

Table 3. Overrepresentation analysis (gene ontology, biological processes) of predicted dog targets of D. immitis L3 and L4-derived miRNAs.

\begin{tabular}{|c|c|c|c|}
\hline \multicolumn{4}{|c|}{ D. immitis L3 Gene Ontology-Biological Processes } \\
\hline Gene Set & Description & $p$-Value & FDR \\
\hline GO:0007389 & $\begin{array}{l}\text { pattern specification } \\
\text { process }\end{array}$ & $5.30 \times 10^{-8}$ & 0 \\
\hline GO:0016579 & $\begin{array}{c}\text { protein } \\
\text { de-ubiquitination }\end{array}$ & $6.47 \times 10^{-8}$ & 0 \\
\hline GO:0000122 & $\begin{array}{c}\text { negative regulation of } \\
\text { transcription by RNA } \\
\text { polymerase II }\end{array}$ & $8.96 \times 10^{-8}$ & 0 \\
\hline GO:0009952 & $\begin{array}{l}\text { anterior/posterior } \\
\text { pattern specification }\end{array}$ & $9.44 \times 10^{-8}$ & 0 \\
\hline GO:0045934 & $\begin{array}{l}\text { negative regulation of } \\
\text { nucleobase- } \\
\text { containing compound } \\
\text { metabolic process }\end{array}$ & $1.94 \times 10^{-7}$ & 0 \\
\hline \multicolumn{4}{|c|}{ D. immitis L4 Gene Ontology-Biological Processes } \\
\hline Gene Set & Description & $p$-Value & FDR \\
\hline GO:0015672 & $\begin{array}{c}\text { monovalent inorganic } \\
\text { cation transport } \\
\text { cation }\end{array}$ & $4.65 \times 10^{-8}$ & 0 \\
\hline GO:0098655 & $\begin{array}{l}\text { transmembrane } \\
\text { transport }\end{array}$ & $7.67 \times 10^{-8}$ & 0 \\
\hline GO:0098662 & $\begin{array}{l}\text { inorganic cation } \\
\text { transmembrane } \\
\text { transport }\end{array}$ & $7.84 \times 10^{-8}$ & 0 \\
\hline GO:0000165 & MAPK cascade & $1.26 \times 10^{-7}$ & 0 \\
\hline GO:0071804 & $\begin{array}{l}\text { cellular potassium } \\
\text { ion transport }\end{array}$ & $2.67 \times 10^{-11}$ & $4.45 \times 10^{-8}$ \\
\hline
\end{tabular}

\section{Discussion}

Our objective was to assess the consistency of the excretory/secretory miRNA profiles of L3 and L4 of two D. immitis strains in vitro, despite their different macrocylic lactone susceptibility status. Overall, we found a good agreement between the current study and previously published data for $D$. immitis [16], although there was generally an even higher agreement between L3 and L4 secretions from the same study, highlighting the impact 
of the inherent inter-experimental variation on resulting data. While the most abundant miRNA species overlapped substantially, their relative rank varied slightly. Regardless of the macrocyclic lactone susceptibility status of the two strains we have examined, our data suggest that the strain involved will have little impact on the most abundantly secreted miRNAs. There was not a dramatic impact of the two D. immitis laboratorial strains on the miRNA species we found, as only two miRNAs were significantly differentially expressed (bma-miR-228 and PC-5p-7293_584, by less than two-fold). Similarly, only four sequences appeared differentially expressed between L3- and L4-derived material (bma-miR-87a_R+1, bma-miR-5866, hpo-miR-10078-5p_1ss10TC, and asu-let-7-5p).

We have sequenced two candidate miRNA markers that are highly abundant and consistently found in both stages and both strains of D. immitis assessed in the study, but also that have been previously found in L3, L4, mf, and adults: PC-3p-5433_825 and PC-5p-7293_584. At the time of writing, these candidate markers did not have any match in miRBase [20,21] or MirGeneDB 2.0 [22], and hence, have no known mammalian orthologues. In addition, these sequences have not been detected in the secretions of other filarial nematodes in vivo thus far. Therefore, we believe that these miRNAs are potentially unique to $D$. immitis. The fact that these two predicted candidates have been found in the secretions of different $D$. immitis strains and across several independent studies speaks in favor of their actual existence (i.e., they are no artifacts). In addition, PC-3p-5433_825 was also discovered in the blood of heartworm-infected dogs, as well as in all D. immitis stages examined thus far [16]. The fact that both candidates were not detected in the somatic miRNA fraction may be due to the different computational approaches to search for novel miRNA candidates [19]. Both sequences were described in adult secretions in $>100$ copies; the sequence similar to PC-5p-7293_584 (PC-5p-263_4142) was detected abundantly in adult female supernatants (>1200 copies), however, with one base difference at the $5^{\prime}$ end.

Within the canine host, L3/L4-specific miRNAs may be detectable as early as 2 weeks post-infection when L3s are molting to L4 in the subcutaneous tissues. However, it is unknown if these would remain detectable after 60-70 days post-infection when most L4s should have molted to the young adult stage (L5) and may be already present in the pulmonary arteries and heart [6]. An important follow-up step will be to assess the temporal changes of $D$. immitis-derived miRNA profiles in the biofluids of experimentally infected dogs through the course of infection. This will allow us to start addressing potential overlaps or discrepancies between in vitro and in vivo secretions; it is currently unclear how well miRNA secretion profiles produced in vitro actually reflect in vivo 'natural' settings.

The detection of miRNA from L3 and L4 represents a challenge due to the small nematode biomass they represent relative to host tissues, their unresolved localization in the hosts' organism, and their rapid development to the following developmental stages. Nevertheless, nematode-derived miRNAs of related filarioid nematodes whose neither adult nor larval stages reside in the hosts' circulatory system have been detected in biofluids. This has been the case for tissue-dwelling filarial nematodes, O. volvulus, $O$. ochengi, and L. loa [11-13], all of which belong to the same family, Onchocercidae, as $D$. immitis. Circulating miRNAs are believed to be either stabilized in protein complexes or packaged in extracellular vesicles (e.g., in exosomes). In both cases, these miRNAs are highly stable and protected from enzymatic degradation or $\mathrm{pH}$ incompatibilities - a good characteristic for diagnostic biomarkers [23,24].

Lagatie and colleagues attempted to detect an array of $O$. volvulus-derived miRNAs in biofluids of naturally infected humans [25]. They concluded that circulating parasite miRNAs are not present at a sufficiently high level to be used as a biomarker for $O$. volvulus infection or treatment monitoring using LNA-based RT-qPCR analysis, as only some miRNAs showed detectable signals in some but not all samples [25]. While the analysis relied on a maximum volume of $200 \mu \mathrm{L}$ serum or plasma, we argue that larger sample volumes may easily be acquired from dogs to be used for diagnosing $D$. immitis infections. In a previous study, several $\mathrm{ml}$ of blood were collected from $\mathrm{mf}$-circulating mature $D$. immitis infections where dog serum had been processed for the detection of 
two D. immitis-specific miRNAs by RT-qPCR [9]. Custom-designed stem-loop RT-qPCR protocols may prove highly efficient to discriminate highly similar miRNA sequences and improve sensitivity [26]. While most studies on parasite-circulating miRNAs have focused on cell-free biofluids, it may be necessary to include the surrounding cell populations (such as phagocytes). In the Litomosoides sigmodontis mouse model, macrophages were shown to represent a parasite miRNA-enriched in vivo reservoir [27]. Generally, there is a need for comparative studies of miRNA profiles of different biofluids of dogs infected with $D$. immitis, as it could help to determine the usefulness of different sample types.

Many immune-related dog pathways are predicted to be targeted by the D. immitis L3 and L4 secreted array of miRNAs. These differ for L3 and L4 secretions and will be worth exploring further for insight into the interactions of larval stages with the canine host. Enriched GO terms resulting from L3 host target predictions revealed several pattern specification processes, which are broader networks involved in cell and organism differentiation and fate/commitment. Horizontal miRNA transfer from parasite to host has been shown; miRNA-driven gene downregulation may have an obvious impact on the host immune response among other fundamental biological processes [17,28,29]. Protein deubiquitination is a conserved mechanism to rescue proteins from degradation or to end ubiquitin signaling [30,31]. It is widely accepted that ubiquitination and deubiquitination are involved in various aspects of immune regulation, encompassing TCR signaling, $\mathrm{T}$ cell differentiation, immune tolerance, and so forth [32]. The accumulating evidence suggests potential interactions between so-called 'immune checkpoint pathways' and ubiquitination/deubiquitination in disease, including in the context of infectious diseases, for example, in bacterial or protozoal (Trypanosoma) infections [31,33]. Several members of the fibroblast growth factor (FGF) and FGF receptor family, among others, are potential targets of L3- and L4-derived miRNAs. These fulfill important roles in cell signaling and in regulating the expression of genes involved in cell growth, proliferation, differentiation, and longevity [34]. MAPKs are involved in various cellular responses, including the production of proinflammatory cytokines or antigen presentation. In helminth infections, signaling through toll-like receptors is mediated through the MAPK cascade; MAPK pathway and ERK1/2 activation support the induction of regulatory T cells/T helper 2 induction [8]. Some parasitic nematode-derived proteins, such as the Acanthocheilonema viteae cystatin have been shown to act as negative regulators of MAPK via other molecules, inducing regulatory responses [35]. Similarly, many bacterial pathogens have been shown to target crucial branches of the MAPK signaling in order to put pressure on the host [36]. Overall, upon uptake by host cells, D. immitis-derived miRNAs have the potential to modulate host immune responses, according to the current computational predictions. These would require experimental validation of the predicted targets in order to represent conclusive results.

\section{Materials and Methods}

\subsection{Mosquito Rearing}

For each mosquito cycle, only quality mosquito eggs of Aedes aegypti (Liverpool strain) were selected to propagate consequent mosquito cycles in an environmental chamber. The mosquito larvae were fed larvae food daily until pupation. The pupae were sorted by sex, discarding roughly $80 \%$ of the males; the majority of the female pupae were placed into rearing buckets.

\subsection{D. immitis Infection for Obtaining L3s and L4s}

The adult female mosquitoes were fed $D$. immitis-infected blood for a minimum of 2.5 h. The mosquitoes were dissected for L3 collection 14 days after blood feeding. The $2 D$. immitis strains used were Missouri-2005 (susceptible to macrocyclic lactones) and Yazoo2013 (resistant to macrocyclic lactones) [37,38]. These 2 strains were chosen to represent the different macrocyclic lactone susceptibility and were based on their availability in the laboratory. 


\subsection{In Vitro Culturing of L3s and L4s}

Batches of approximately $250 \mathrm{D}$. immitis L3s were washed a minimum of five times with fresh RPMI to completely remove mosquito debris. Fresh culture media was added to well plates and L3s were added into trans-well plates and incubated in a $5 \% \mathrm{CO}_{2}$ incubator at $37^{\circ} \mathrm{C}$. The supernatant was collected every $48 \mathrm{~h}$ for two weeks. The supernatant collected on days 2 and 4 of culture were designated as L3-derived supernatants; those collected on days 8 and 12 were L4-derived supernatants. After each supernatant collection, dead L3/L4s were removed and new sterile media were added to the remaining live worms. In total, there were 3 experimental replicates per larval stage and strain (Table 4). All supernatant samples were stored at $-80^{\circ} \mathrm{C}$ until further processing.

Table 4. Experimental replicates and cultured larvae.

\begin{tabular}{ccc}
\hline Experimental Replicate & D. immitis Strain & Number of L3 Cultured \\
\hline 1 & Yazoo & 3640 \\
2 & Yazoo & 1560 \\
3 & Yazoo & 1560 \\
4 & Missouri & 3120 \\
5 & Missouri & 1560 \\
6 & Missouri & 780 \\
\hline
\end{tabular}

\subsection{RNA Extraction}

Total RNA was extracted from L3- and L4-derived supernatant of each strain, in triplicate, using the Norgen plasma/serum RNA purification midi kit (Norgen Biotek Corp., Thorold, ON, Canada) [16]. The RNA was extracted from $1 \mathrm{~mL}$ of each supernatant sample, eluted in $25 \mu \mathrm{L}$, and stored at $-80^{\circ} \mathrm{C}$. To avoid cross-contamination, the supernatants of 2 different strains were never extracted alongside each other.

\subsection{RNA Sequencing and Analysis}

There were 6 samples from each strain, encompassing 3 replicates from L3 and 3 from L4 (Table 4), submitted to miRNA deep sequencing. The sequence input for sequencing was limited to the 18-30 nt size range. FastQC comprised a per-base sequence quality assessment for each submitted sample (not shown). The general characteristics of the reads (e.g., read length distribution) obtained for the different libraries were assessed by LC Sciences. The raw data were filtered using a filter module in an in-house program to delete low-quality reads, $3^{\prime}$ adapter sequences, and contaminations. The sequences $\geq 18 \mathrm{nt}$ of clean data were annotated in the Rfam database (http:/ / www.sanger.ac.uk/software/Rfam) [39] to remove non-coding RNA (rRNA, tRNA, snRNA, snoRNA) and degradation fragments of mRNA. The remaining sequences were aligned against all nematode miRNAs listed in miRbase (Release 21, http:/ / www.miRbase.org) [20,21]; perfectly matched sequences were considered conserved $D$. immitis miRNAs. Nematode species, for which miRNAs are available in miRNAse, are the following: Brugia malayi, Ascaris suum, Haemonchus contortus, Heligmosomoides polygyrus, Strongyloides ratti, Caenorhabditis elegans, C. briggsae, C. brenneri, C. remanei, Pristionchus pacificus, and Panagrellus redivivus. Reads were then assigned to groups reflecting the confidence level that these sequences are truly $D$. immitis miRNAs. These groups are based on genome mapping properties, secondary structure, and homology to known miRNAs. Group 1: the reads were mapped to miRNAs/pre-miRNAs of specific species in miRbase, and the pre-miRNAs were further mapped to a genome and EST; Group 2a: the reads were mapped to miRNAs/pre-miRNAs of selected species in miRbase, and the mapped pre-miRNAs were not further mapped to a genome, but the reads (and, of course, the miRNAs of the pre-miRNAs) were mapped to a genome. The extended genome sequences from the genome loci may form hairpins; Group 2b: the reads were mapped to miRNAs/pre-miRNAs of selected species in miRbase and the mapped pre-miRNAs were not further mapped to a genome, but the reads (and, of course, the miRNAs of the premiRNAs) were mapped to a genome. The extended genome sequences from the genome 
loci may not form hairpins; Group 3: the reads were mapped to miRNAs/pre-miRNAs of selected species in miRbase and the mapped pre-miRNAs were not further mapped to a genome, and the reads were not mapped to a genome, either, but the reads were mapped to the mature miRNAs; Group 4 (predicted candidates): the reads were not mapped to pre-miRNAs of selected species in miRbase, but the reads were mapped to a genome, and the extended genome sequences from the genome may form hairpins. The normalization of sequence counts from each sample (or dataset) was achieved by dividing the counts by a library size parameter of the corresponding sample. The library size parameter is a median value of the ratio between the counts of a specific sample and a pseudo-reference sample. A count number in the pseudo-reference sample is the count geometric mean across all samples (see [16]).

\subsection{Predicted miRNA Targets and Pathway Enrichment}

miRNA target prediction was performed using the standalone TargetScan software package v. 7 (http:/ / www.targetscan.org) [40], implemented as a standalone workflow under iPortal [41] and openBIS [42], using default parameters. All host genes that were targeted at least twice, regardless of the context scores, were used in enrichment analyses. The $R$ version of the WEB-based Gene SeT Analysis Toolkit (WebGestalt) [43,44] was employed for overrepresentation analysis (ORA) based on gene ontology "biological processes" [45], using default parameters, with FDR $<0.05$. As a reference gene list, we employed the immunological signatures (collection C7) from the Molecular Signatures Database v 6.2 (Gene Set Enrichment Analysis database, http:/ / software.broadinstitute. org/gsea/msigdb/index.jsp) [46,47].

\section{Conclusions}

Mature miRNA sequences shed by L3 and L4 overlapped largely, both qualitatively and quantitatively. Similarly, the laboratorial D. immitis strain did not significantly impact the excretory/secretory miRNA profile in vitro, regardless of their macrocyclic lactone susceptibility status. The presence and temporal expression of the candidate miRNA biomarkers in vivo in biofluids of the canine host should be further investigated. The potential of L3- and L4-specific sequences as biomarkers of early heartworm infection remains to be assessed and validated in vivo using samples derived from experimentally and naturally infected dogs. Nevertheless, there are challenges associated with the limited biomass of such larval stages and sensitivity issues of the currently available miRNA detection assays.

Supplementary Materials: The following are available online at https:/ / www.mdpi.com/article/10 .3390/pathogens10070786/s1: Table S1. miRNAs shed by two D. immitis strains, L3 and L4 stages in vitro; Table S2. miRNAs shed by D. immitis L3 in vitro (stratified by strain); Table S3. miRNAs shed by $D$. immitis L4 in vitro (stratified by strain); Table S4. miRNAs shed by D. immitis strain Yazoo in vitro (stratified by stage); Table S5. miRNAs shed by D. immitis strain Missouri in vitro (stratified by stage).

Author Contributions: Conceptualization, L.T., E.J.B., T.C. and G.G.V.; parasite culture, E.J.B.; L.T. bioinformatics analysis, L.T.; data analysis, L.T., E.J.B., T.C. and G.G.V.; writing-original draft preparation, L.T. and G.G.V. All authors have read and agreed to the published version of the manuscript.

Funding: This research was funded by Zoetis Inc.

Institutional Review Board Statement: The study was conducted according to the guidelines of the Declaration of Helsinki, and approved by the Institutional Review Board (or Ethics Committee) of the University of Georgia (A2016 10-006, 17 November 2017).

Informed Consent Statement: Not applicable.

Data Availability Statement: The raw data reads were deposited in Gene Expression Omnibus (GEO; http:/ / www.ncbi.nlm.nih.gov/geo/) under the accession number GSE174744. 
Acknowledgments: We would like to thank Zoetis Inc. for providing funding and the NIH-NIAID Filariasis Research Reagent Resource Center (FR3) for providing the biological materials for the completion of this project. We are grateful for the laboratory assistance provided by Justin Cueva.

Conflicts of Interest: L.T., E.J.B. and G.G.V. have received research grants from Zoetis Inc. T.C. is employed by Zoetis Inc., and E.J.B. is currently employed by Boehringer Ingelheim Animal Health The funders had no role in the design of the study; in the collection, analyses, or interpretation of data; in the writing of the manuscript, or in the decision to publish the results.

\section{References}

1. McCall, J.W.; Genchi, C.; Kramer, L.H.; Guerrero, J.; Venco, L. Heartworm Disease in Animals and Humans. Adv. Parasitol. 2008, 66, 193-285. [CrossRef]

2. Bowman, D.D.; Atkins, C.E. Heartworm Biology, Treatment, and Control. Vet. Clin. N. Am. Small Anim. Pract. 2009, 39, 1127-1158. [CrossRef] [PubMed]

3. Drake, J.; Wiseman, S. Increasing Incidence of Dirofilaria immitis in Dogs in USA with Focus on the Southeast Region $2013-2016$. Parasit. Vectors 2018, 11, 39. [CrossRef] [PubMed]

4. Nelson, C.T.; McCall, J.W.; Carithers, D. Current Canine Guidelines for the Prevention, Diagnosis, and Management of Heartworm (Dirofilaria immitis) Infection in Dogs; American Heartworm Society: Wilmington, DE, USA, 2018.

5. Atkins, C.E. Comparison of Results of Three Commercial Heartworm Antigen Test Kits in Dogs with Low Heartworm Burdens. J. Am. Vet. Med. Assoc. 2003, 222, 1221-1223. [CrossRef]

6. Kotani, T.; Powers, K.G. Developmental Stages of Dirofilaria immitis in the Dog. Am. J. Vet. Res. 1982, 43, 2199-2206. [PubMed]

7. McSorley, H.J.; Hewitson, J.P.; Maizels, R.M. Immunomodulation by Helminth Parasites: Defining Mechanisms and Mediators. Int. J. Parasitol. 2013, 43, 301-310. [CrossRef]

8. Zakeri, A.; Hansen, E.P.; Andersen, S.D.; Williams, A.R.; Nejsum, P. Immunomodulation by Helminths: Intracellular Pathways and Extracellular Vesicles. Front. Immunol. 2018, 9, 2349. [CrossRef]

9. Tritten, L.; Burkman, E.; Moorhead, A.; Satti, M.; Geary, J.; Mackenzie, C.; Geary, T. Detection of Circulating Parasite-Derived MicroRNAs in Filarial Infections. PLoS Negl. Trop. Dis. 2014, 8, e2971. [CrossRef]

10. Tritten, L.; O’Neill, M.; Nutting, C.; Wanji, S.; Njouendoui, A.; Fombad, F.; Kengne-Ouaffo, J.; Mackenzie, C.; Geary, T. Loa loa and Onchocerca ochengi miRNAs Detected in Host Circulation. Mol. Biochem. Parasitol. 2014, 198, 14-17. [CrossRef]

11. Hoy, A.M.; Lundie, R.J.; Ivens, A.; Quintana, J.F.; Nausch, N.; Forster, T.; Jones, F.; Kabatereine, N.B.; Dunne, D.W.; Mutapi, F.; et al. Parasite-Derived MicroRNAs in Host Serum as Novel Biomarkers of Helminth Infection. PLoS Negl. Trop. Dis. 2014, 8, e2701. [CrossRef]

12. Quintana, J.F.; Makepeace, B.L.; Babayan, S.A.; Ivens, A.; Pfarr, K.M.; Blaxter, M.; Debrah, A.; Wanji, S.; Ngangyung, H.F.; Bah, G.S.; et al. Extracellular Onchocerca-Derived Small RNAs in Host Nodules and Blood. Parasit. Vectors 2015, 8, 58. [CrossRef]

13. Zamanian, M.; Fraser, L.M.; Agbedanu, P.N.; Harischandra, H.; Moorhead, A.R.; Day, T.A.; Bartholomay, L.C.; Kimber, M.J. Release of Small RNA-Containing Exosome-like Vesicles from the Human Filarial Parasite Brugia malayi. PLoS Negl. Trop. Dis. 2015, 9, e0004069. [CrossRef] [PubMed]

14. Quintana, J.F.; Babayan, S.A.; Buck, A.H. Small RNAs and Extracellular Vesicles in Filarial Nematodes: From Nematode Development to Diagnostics. Parasite Immunol. 2016, 39, e12395. [CrossRef] [PubMed]

15. Tritten, L.; Geary, T.G. MicroRNAs of Filarial Nematodes: A New Frontier in Host-Pathogen Interactions. In Non-Coding RNAs and Inter-Kingdom Communication; Springer International Publishing: Berlin/Heidelberg, Germany, 2016; ISBN 978-3-319-39494-7.

16. Tritten, L.; Clarke, D.; Timmins, S.; McTier, T.; Geary, T.G. Dirofilaria immitis Exhibits Sex- and Stage-Specific Differences in Excretory/Secretory miRNA and Protein Profiles. Vet. Parasitol. 2016, 232, 1-7. [CrossRef] [PubMed]

17. Buck, A.H.; Coakley, G.; Simbari, F.; McSorley, H.J.; Quintana, J.F.; Le Bihan, T.; Kumar, S.; Abreu-Goodger, C.; Lear, M.; Harcus, Y.; et al. Exosomes Secreted by Nematode Parasites Transfer Small RNAs to Mammalian Cells and Modulate Innate Immunity. Nat. Commun. 2014, 5, 5488. [CrossRef] [PubMed]

18. Kim, H.; Kim, J.; Kim, K.; Chang, H.; You, K.; Kim, V.N. Bias-Minimized Quantification of microRNA Reveals Widespread Alternative Processing and 3' End Modification. Nucleic Acids Res. 2019, 47, 2630-2640. [CrossRef]

19. Fu, Y.; Lan, J.; Wu, X.; Yang, D.; Zhang, Z.; Nie, H.; Hou, R.; Zhang, R.; Zheng, W.; Xie, Y.; et al. Identification of Dirofilaria immitis miRNA Using Illumina Deep Sequencing. Vet. Res. 2013, 44, 3. [CrossRef] [PubMed]

20. Griffiths-Jones, S.; Grocock, R.J.; van Dongen, S.; Bateman, A.; Enright, A.J. MiRBase: microRNA Sequences, Targets and Gene Nomenclature. Nucleic Acids Res. 2006, 34, D140-D144. [CrossRef]

21. Griffiths-Jones, S.; Saini, H.K.; van Dongen, S.; Enright, A.J. MiRBase: Tools for microRNA Genomics. Nucleic Acids Res. 2008, 36, D154-D158. [CrossRef]

22. Fromm, B.; Domanska, D.; Høye, E.; Ovchinnikov, V.; Kang, W.; Aparicio-Puerta, E.; Johansen, M.; Flatmark, K.; Mathelier, A.; Hovig, E.; et al. MirGeneDB 2.0: The Metazoan microRNA Complement. Nucleic Acids Res. 2020, 48, D132-D141. [CrossRef]

23. Turchinovich, A.; Weiz, L.; Langheinz, A.; Burwinkel, B. Characterization of Extracellular Circulating microRNA. Nucleic Acids Res. 2011, 39, 7223-7233. [CrossRef]

24. Wang, K.; Zhang, S.; Weber, J.; Baxter, D.; Galas, D.J. Export of microRNAs and microRNA-Protective Protein by Mammalian Cells. Nucleic Acids Res. 2010, 38, 7248-7259. [CrossRef] 
25. Lagatie, O.; Batsa Debrah, L.; Debrah, A.; Stuyver, L.J. Plasma-Derived Parasitic microRNAs Have Insufficient Concentrations to Be Used as Diagnostic Biomarker for Detection of Onchocerca volvulus Infection or Treatment Monitoring Using LNA-Based RT-QPCR. Parasitol. Res. 2017, 116, 1013-1022. [CrossRef]

26. Chen, C.; Ridzon, D.A.; Broomer, A.J.; Zhou, Z.; Lee, D.H.; Nguyen, J.T.; Barbisin, M.; Xu, N.L.; Mahuvakar, V.R.; Andersen, M.R.; et al. Real-Time Quantification of microRNAs by Stem-Loop RT-PCR. Nucleic Acids Res. 2005, 33, e179. [CrossRef]

27. Quintana, J.F.; Kumar, S.; Ivens, A.; Chow, F.W.N.; Hoy, A.M.; Fulton, A.; Dickinson, P.; Martin, C.; Taylor, M.; Babayan, S.A.; et al. Comparative Analysis of Small RNAs Released by the Filarial Nematode Litomosoides sigmodontis in Vitro and in Vivo. PLoS Negl. Trop. Dis. 2019, 13, e0007811. [CrossRef]

28. Tritten, L.; Geary, T.G. Helminth Extracellular Vesicles in Host-Parasite Interactions. Curr. Opin. Microbiol. 2018, 46, 73-79. [CrossRef]

29. Eichenberger, R.M.; Sotillo, J.; Loukas, A. Immunobiology of Parasitic Worm Extracellular Vesicles. Immunol. Cell Biol. 2018. [CrossRef] [PubMed]

30. Micel, L.N.; Tentler, J.J.; Smith, P.G.; Eckhardt, G.S. Role of Ubiquitin Ligases and the Proteasome in Oncogenesis: Novel Targets for Anticancer Therapies. J. Clin. Oncol. Off. J. Am. Soc. Clin. Oncol. 2013, 31, 1231-1238. [CrossRef] [PubMed]

31. Liu, J.; Cheng, Y.; Zheng, M.; Yuan, B.; Wang, Z.; Li, X.; Yin, J.; Ye, M.; Song, Y. Targeting the Ubiquitination/Deubiquitination Process to Regulate Immune Checkpoint Pathways. Signal Transduct. Target. Ther. 2021, 6, 28. [CrossRef] [PubMed]

32. Zeng, P.; Ma, J.; Yang, R.; Liu, Y.-C. Immune Regulation by Ubiquitin Tagging as Checkpoint Code. Curr. Top. Microbiol. Immunol. 2017, 410, 215-248. [CrossRef] [PubMed]

33. Perrett, C.A.; Lin, D.Y.-W.; Zhou, D. Interactions of Bacterial Proteins with Host Eukaryotic Ubiquitin Pathways. Front. Microbiol. 2011, 2, 143. [CrossRef]

34. Xie, Y.; Su, N.; Yang, J.; Tan, Q.; Huang, S.; Jin, M.; Ni, Z.; Zhang, B.; Zhang, D.; Luo, F.; et al. FGF/FGFR Signaling in Health and Disease. Signal Transduct. Target. Ther. 2020, 5, 181. [CrossRef]

35. Klotz, C.; Ziegler, T.; Figueiredo, A.S.; Rausch, S.; Hepworth, M.R.; Obsivac, N.; Sers, C.; Lang, R.; Hammerstein, P.; Lucius, R.; et al. A Helminth Immunomodulator Exploits Host Signaling Events to Regulate Cytokine Production in Macrophages. PLoS Pathog. 2011, 7, e1001248. [CrossRef] [PubMed]

36. Reddick, L.E.; Alto, N.M. Bacteria Fighting Back: How Pathogens Target and Subvert the Host Innate Immune System. Mol. Cell 2014, 54, 321-328. [CrossRef] [PubMed]

37. Michalski, M.L.; Griffiths, K.G.; Williams, S.A.; Kaplan, R.M.; Moorhead, A.R. The NIH-NIAID Filariasis Research Reagent Resource Center. PLoS Negl. Trop. Dis. 2011, 5, e1261. [CrossRef] [PubMed]

38. Maclean, M.J.; Savadelis, M.D.; Coates, R.; Dzimianski, M.T.; Jones, C.; Benbow, C.; Storey, B.E.; Kaplan, R.M.; Moorhead, A.R.; Wolstenholme, A.J. Does Evaluation of in Vitro Microfilarial Motility Reflect the Resistance Status of Dirofilaria immitis Isolates to Macrocyclic Lactones? Parasit. Vectors 2017, 10, 17-23. [CrossRef]

39. Gardner, P.P.; Daub, J.; Tate, J.; Moore, B.L.; Osuch, I.H.; Griffiths-Jones, S.; Finn, R.D.; Nawrocki, E.P.; Kolbe, D.L.; Eddy, S.R.; et al. Rfam: Wikipedia, Clans and the "Decimal" Release. Nucleic Acids Res. 2011, 39, D141-D145. [CrossRef]

40. Agarwal, V.; Bell, G.W.; Nam, J.-W.; Bartel, D.P. Predicting Effective microRNA Target Sites in Mammalian mRNAs. eLife 2015, 4. [CrossRef]

41. Kunszt, P.; Blum, L.; Hullár, B.; Schmid, E.; Srebniak, A.; Wolski, W.; Rinn, B.; Elmer, F.-J.; Ramakrishnan, C.; Quandt, A.; et al. IPortal: The Swiss Grid Proteomics Portal: Requirements and New Features Based on Experience and Usability Considerations. Concurr. Comput. Pract. Exp. 2015, 27, 433-445. [CrossRef]

42. Bauch, A.; Adamczyk, I.; Buczek, P.; Elmer, F.-J.; Enimanev, K.; Glyzewski, P.; Kohler, M.; Pylak, T.; Quandt, A.; Ramakrishnan, C.; et al. OpenBIS: A Flexible Framework for Managing and Analyzing Complex Data in Biology Research. BMC Bioinform. 2011, 12, 468. [CrossRef]

43. Zhang, B.; Kirov, S.; Snoddy, J. WebGestalt: An Integrated System for Exploring Gene Sets in Various Biological Contexts. Nucleic Acids Res. 2005, 33, W741-W748. [CrossRef]

44. Wang, J.; Vasaikar, S.; Shi, Z.; Greer, M.; Zhang, B. WebGestalt 2017: A More Comprehensive, Powerful, Flexible and Interactive Gene Set Enrichment Analysis Toolkit. Nucleic Acids Res. 2017, 45, W130-W137. [CrossRef]

45. Ashburner, M.; Ball, C.A.; Blake, J.A.; Botstein, D.; Butler, H.; Cherry, J.M.; Davis, A.P.; Dolinski, K.; Dwight, S.S.; Eppig, J.T.; et al. Gene Ontology: Tool for the Unification of Biology. The Gene Ontology Consortium. Nat. Genet. 2000, 25, 25-29. [CrossRef] [PubMed]

46. Liberzon, A.; Subramanian, A.; Pinchback, R.; Thorvaldsdóttir, H.; Tamayo, P.; Mesirov, J.P. Molecular Signatures Database (MSigDB) 3.0. Bioinforma. Oxf. Engl. 2011, 27, 1739-1740. [CrossRef] [PubMed]

47. Liberzon, A.; Birger, C.; Thorvaldsdóttir, H.; Ghandi, M.; Mesirov, J.P.; Tamayo, P. The Molecular Signatures Database (MSigDB) Hallmark Gene Set Collection. Cell Syst. 2015, 1, 417-425. [CrossRef] [PubMed] 